\title{
Efficacy of Combined Herbal Capsule on Sexual Function in Postmenopausal Women: A Triple-Blinded Randomized Controlled Trial
}

\author{
Paria Eliasvandi $^{1 \mathbb{D}}$, Laleh Khodaie ${ }^{2}$, Sakineh Mohammad Alizadeh Charandabi ${ }^{3}$, Mojgan Mirghafourvand ${ }^{*}$
}

\begin{abstract}
Objectives: Considering the prevalence of sexual problems in postmenopausal women as well as complications of hormone therapy, the tendency to use complementary medicine has increased. The present study was conducted to evaluate the efficacy of combined herbal drug on the sexual function of postmenopausal women.

Materials and Methods: The present triple-blinded randomized controlled trial was done on 64 Iranian women in 2017. The women were randomly allocated to 2 groups of intervention (combined herbal capsules users) and control (placebo users). Herbal capsules contained carnation, Anise, Anison, violets, Terminalia chebula, fresh green raisins, Senna leaves, Tangerine and Golqand. Participants in both groups took 2 capsules twice a day (before lunch and before dinner) for 4 weeks. The Female Sexual Function Index (FSFI) was filled out before the intervention and 4 weeks after the intervention.

Results: No statistically significant difference was observed between the study groups in terms of socio-demographic properties, the total mean score of sexual function and its sub-domains before the intervention. Four weeks after the intervention, the total mean (SD) sexual function score was 26.99 (5.31) in the combined herbal capsule group and it was 18.35 (4.51) in the placebo group, so it was significantly higher in the combined herbal capsule group compared to the placebo group (adjusted mean difference $=8.70 ; 95 \% \mathrm{CI}=6.58$ - 10.82). Moreover, there was a significant difference in the mean score of desire, arousal, lubrication, orgasm, satisfaction, and pain subscales between study groups after the intervention.

Conclusions: The results of the study show that taking combined herbal capsules improved sexual function in postmenopausal women. However, further studies are necessary to decide on its use as an alternative medication for the improvement of sexual function.

Keywords: Herbal medicine, Sexual dysfunction, Menopause
\end{abstract}

\section{Introduction}

Sexual function as the couples' interaction is associated with hormonal and neurovascular factors. Several factors such as biological, cultural, and social characteristics and also interpersonal relationships influence sexual function (1). An individual or a couple may experience sexual dysfunction during any stage of sexual activity including sexual desire, arousal, orgasm, and other stages. Sexual dysfunction may have a profound impact on the quality of one's perception of sexual life (2) and is divided into disorders of desire, arousal, orgasm, and pain during intercourse. The rate of sexual disorders in the general population in the world and Iran is reported to be $46 \%$ and $35 \%$, respectively $(3,4)$. Another study conducted in Iran indicated that approximately most (two-thirds) of women suffered from at least one sexual problem during the postmenopausal period (5).

Risk factors for female and male sexual dysfunction include health status, socio-demographic conditions, some chronic diseases such as endocrine, cardiovascular, genital and psychiatric or mental diseases and increasing age. Moreover, the effects of smoking and hormonal factors are well known (3).

Menopause is a physiological period in women's life characterized by 12 months of amenorrhea without the existence of biological and physiological factors (6). During menopause transition and menopause, women may experience hot flashes, sleep disorders, vaginal dryness, urinary incontinence, and sexual dysfunction. Although the average age of menopause is considered to be 51.4, the age and severity of symptoms are influenced by several factors such as genetics, smoking, exercise, nutrition, stress, and hormonal changes (estradiol, folliclestimulating hormone, dehydroepiandrosterone sulfate, testosterone, and androstenedione) (6,7). Menopause is closely related to age and it seems that certain types of sexual dysfunction, such as vaginal dryness and painful intercourse, are constantly increasing due to estrogen deficiency $(8,9)$. In one study, the prevalence of vaginal dryness in the late years of fertility, early and late menopausal transition periods and the first three years after menopause was reported to be $3 \%, 4 \%, 21 \%$, and 
$47 \%$, respectively $(8,10)$. Additionally, low sexual desire and arousal are reported to be higher in postmenopausal women than in other groups $(8,9)$.

The usual treatment for reducing menopausal complications is hormone replacement therapy (HRT). Studies have shown that estrogen alone or along with progesterone has a low to moderate effect on the sexual performance in women during menopause transition and menopause (11). HRT in postmenopausal women is associated with complications such as increased incidence of non-cardiac events, pulmonary embolism and deep vein thrombosis, stroke, increased prevalence of gallstones and increased risk of breast and endometrial cancer (6).

In recent years, complementary medicine has been frequently used in treating menopausal complications. Evidence indicates that different pharmacologic and -non-pharmacologic treatments are used for treating sexual dysfunction (8). One of these treatments is the use of herbal medicines (12).

Phytoestrogens are plant compounds with estrogen-like properties that are used to treat menopausal complications $(13,14)$. According to studies, phytoestrogens can protect against the development of cancers, especially hormonerelated cancers including breast and endometrial cancers (15). In this study, a combined herbal capsule containing cloves (Syzygium aromaticum), Roman anemones or Anise, green anise or fennel, green raisin (Vitis vinifera), Alhagi maurorum, Viola sp, yellow hill and Senna combined with Golqand (rose petals and honey) was used (16). S. aromaticum has antioxidant, antimicrobial and antifungal properties (17). Anise and fennel, with the botanical names Pimpinella anisum and Foeniculum vulgare, are expected to improve sexual function, reduce dyspareunia and vaginal dryness due to phytoestrogenic effects $(18,19)$. Based on traditional medicine resources, raisin increases libido and honey improves sexual function by creating a sense of happiness $(20,21)$. Other plants in the combination such as A. maurorum, Viola sp, yellow hill, and Senna are regulators that help reduce the complications and increase the effect of herbal combination (16).

Regarding the prevalence of sexual problems due to atrophic vaginitis caused by hypoestrogenemia during menopause (8) and the interest of women in the use of complementary medicines (22), investigating the effects of herbal drugs especially phytoestrogens is necessary. Therefore, the present study was conducted to evaluate the efficacy of combined herbal capsules on the sexual function of postmenopausal women. This research is the first study to determine the effect of a combined herbal drug on sexual function in women.

\footnotetext{
Materials and Methods

Study Design and Participants

The present study was a triple-blinded randomized controlled trial (data collector, participants, and data analyzer were blinded to the type of intervention received)
}

which was done on 64 married postmenopausal women who referred to community health centers in Kermanshah, Iran, in 2017.

The inclusion criteria consisted menopausal women, aged between 40 and 60 years, no allergy to the herbal compounds used in this research, women living with their husbands, being literate, having a phone number and willingness to participate in the study. The exclusion criteria included suffering from neuromuscular disorders (multiple sclerosis, stroke, and Parkinson disease) and gastrointestinal diseases including colorectal cancer, inflammation, diarrhea, and duct stenosis, taking medications such as opiates, antidepressants, calcium channel blockers, ibuprofen, anticoagulants, diuretics, iron and calcium supplements, and receiving hormone therapy.

Based on a study by Nazarpour et al (23), considering the mean pre-intervention sexual function score $\left(m_{1}=24.11\right)$ and assuming a $20 \%$ increase in the postintervention mean score of sexual function $\left(\mathrm{m}_{2}=28.93\right)$, one-sided $\alpha=0.05, \beta=0.1, \mathrm{SD}_{1}=\mathrm{SD}_{2}=6.04$, the sample size calculation showed that 28 people were needed in each group. However, by taking into account the loss to followup, the final sample size was considered to be 32 in each group.

\section{Sampling}

The sampling started after obtaining the approval from the Ethics Committee of Tabriz University of Medical Sciences (Ethics Code: IR.TBZMED.REC.1395.281) and registering it in the Iranian Registry of Clinical Trials (identifier: IRCT2016043010324N32; https:/www.irct. $\mathrm{ir} /$ trial/10807). A total of 23 urban community health centers were located in Kermanshah. These centers were public, governmental, or private first-level referral centers. Four centers with different socioeconomic levels were chosen, from which the participants were selected by means of convenience sampling. At first, by referring to the health records and documents of the centers and considering the inclusion criteria, some people were selected and subsequently contacted to refer to the health centers. Then, the checklists of inclusion and exclusion criteria were completed through interviews with women. After explaining the goals of the study and obtaining an informed consent form, the women who were eligible to enter the study completed socio-demographic characteristics questionnaire and the Female Sexual Function Index (FSFI). Four weeks after the intervention, the FSFI questionnaire was completed by interviewing the participants.

\section{Random Allocation and Intervention}

Women were randomly allocated to 2 groups, including a treatment group receiving combined herbal capsule and a control group receiving placebo, using block randomization method with block sizes of 4 and 6 and 
an allocation ratio of 1:1. The medication and placebo to be used for 2 weeks were placed in identical small packs which were numbered sequentially. Both small packs with the same content were placed inside larger packs. The large pack and the packs inside it received the same code. The packs were opened in the order of the entry of the individuals to the study, and a small pack to be used for 2 weeks was delivered to participants by the researcher. At the end of the second week, the participants were revisited and the second small packs containing either a drug or a placebo was delivered. The completed drug intake checklist for the first 2 weeks was also received.

The capsules containing the drug and the placebo were prepared in a completely identical appearance. There was no need to prepare herbarium code for already known and regularly-used herbs. They were purchased from herbal market. The veracity of the compounds was confirmed by a pharmacognosist and an herbalist and they were powdered by mill. Then, they were combined based on their specific proportions (carnation: 4 units, Anise and Anison: 6 units of each, violets: 12 units, Terminalia chebula and fresh green raisins: 25 units of each, Senna leaves, tranbinbine, and Golqand: 50 units of each). For preparing the capsule, firstly, A. maurorum, raisin and Golqand were blended. Secondly, flower buds of Syzygium aromaticum, fruits of Pimpinella anisum, flowers of Viola odorata, fruits of Terminalia chebula, and leaves of Cassia angustifolia were fully ground and sieved. Thirdly, all the ingredients were blended and completely dried. Afterwards, they were filled in $500 \mathrm{mg}$ capsule shells. The process started in a way that tranbinbine was milled and mixed up with Golqand, and was kept in that condition for few hours to spread the moisture of Golqand to tranbinbine. Then, Golqand was dried with raisins in hay . Other ingredients were powdered and mixed with Golqand and raisins. Then the herbal combination was examined for microbial contamination in the microbial control laboratory. In order to eliminate the microbes, after placing the prepared herbal powder in an oven with a temperature of $50^{\circ} \mathrm{C}$ and starch powder in an oven with a temperature of $80^{\circ} \mathrm{C}$. Total aerobic microbial count was determined for both samples. In this test, the number of microbes was less than $1000 \mathrm{CFU} / \mathrm{g}$ (colony forming units per gram). There were no Escherichia coli and Salmonella bacteria in any of the tested specimens. According to the guidelines of United States Pharmacopeia (USP), the combined herbal powder and starch were both at an acceptable level for microbial control. The capsules were standardized for the presence of the active ingredient, based on the marker compounds used in the herbs. In order to prepare the placebo, $500 \mathrm{mg}$ capsules containing starch were used. The participants were required to take 2 capsules twice a day (before lunch and before dinner) for 4 weeks. Given that food prevents the mentioned medicinal plants to function, they were taken before meals for better function. Usually, gastrointestinal medicines are taken before meals. Participants were contacted once a week to ensure participants' consumption.

\section{Instruments}

In the present study, socio-demographic characteristics questionnaire and FSFI were used for data collection. The socio-demographic characteristics questionnaire included questions about age, occupation, the income sufficiency, education, husband's education, housing status, weight, and height. Content and face validity were used for determining the validity and the questionnaire was sent to 10 professors of Tabriz University of Medical Sciences and their comments were applied.

FSFI was developed by Rosen et al (24) to evaluate women's sexual function. It is a valid questionnaire for assessing sexual function during the past 4 weeks. The questionnaire contains 19 questions, which evaluates 6 different domains including desire, arousal, lubrication, orgasm, satisfaction, and pain. For each of the answers to questions $3-14,17-19,0-5$ points and for questions 1 , $2,15,16,1-5$ points were considered. The scores for each domain were obtained from questions in each domain and then multiplied by the coefficient of each domain (desire 0.6 , arousal and lubrication 0.3 , orgasm, satisfaction and pain 0.4 ). The libido score ranged from 1.2 to 6 points, and the scores of other domains ranged from 0 to 6 points. The total score, which was computed from the sum of 6 domains, ranged between 2 and 36. Higher scores show better sexual function. A total sexual function score of 26 or less is considered to be sexual dysfunction (20). This scale was validated by Mohammadi et al on 53 women with a sensitivity of $82 \%$ and $83 \%$ in Tehran in 2008 (21).

\section{Statistical Analysis}

SPSS version 21.0 (IBM Crop., Armonk, N.Y., USA) was used for data analysis. Kolmogorov-Smirnov test was used to evaluate the normal distribution of quantitative data, indicating that all data had a normal distribution. To compare the consistency of the 2 groups, independent $t$ test, Fisher exact test and chi-square test were used. To compare the total score of sexual function and its dimensions before and after the intervention, independent $t$ test and ANCOVA were used, respectively. At all stages, $P<0.05$ was considered statistically significant. All analyses were performed on the basis of intention-to-treat.

\section{Results}

The sampling process was conducted in 2017 from February to July. During this period, 122 women were examined for inclusion criteria. Of these, 39 were excluded due to the lack of eligibility criteria and 19 were excluded due to lack of willingness to participate. A total of 64 women were enrolled in the study, 32 of whom were assigned to the group receiving herbal capsules and 32 women to the placebo group who received the relevant intervention. Socio-demographic questionnaire and 
FSFI were completed by interviewing the participant. Participants were followed up for 4 weeks. One of the placebo recipients refused to continue the intervention at the end of the first week, but his follow-up continued until the end of the fourth week. The FSFI questionnaire was completed by interviewing the participants at the end of the fourth week. Finally, data analysis was performed on the data obtained from 32 women in the treatment group and 32 women in the placebo group (Figure 1).

The socio-demographic data of the participants were presented in Table 1. No significant difference was observed between the 2 groups in relation to sociodemographic data. The mean age (mean \pm standard deviation [SD]) of women in the drug group was $50.53 \pm 4.78$ and it was $52.34 \pm 4.50$ years old in the placebo group. Most of the participants $(84.4 \%$ in the drug group and $87.5 \%$ in the placebo group) were housewives. More than half of the participants $(56.3 \%$ in the drug group and $53.1 \%$ in the placebo group) had elementary education. The majority of participants $(96.9 \%$ of the drug group and $90.6 \%$ of the placebo group) reported their economic situation as favorable and relatively favorable. The mean
(SD) body mass index (BMI) was $27.67 \pm 4.16$ in the drug group and it was $27.83 \pm 3.61$ in the placebo group. Most of the participants had three maternal experiences $(40.6 \%$ in the drug group, $31.3 \%$ in the placebo group) and more than three births $(25.2 \%$ in the drug group and $43.8 \%$ in the placebo group). About half of participants (48.4\% in the drug group and $59.4 \%$ in the placebo group) reported their health status as average.

The total mean (SD) FSFI score before the intervention was $17.46 \pm 5.19$ in the drug group and it was $17.56 \pm 3.22$ in the placebo group, and the difference was significant based on independent $t$ test. No statistically significant difference was observed between the 2 groups before the intervention $(P=0.927)$. However, the total mean (SD) sexual function score after the intervention was $26.99 \pm 5.31$ in the combined herbal capsule group and it was $18.35 \pm 4.51$ in the placebo group. According to analysis of the covariance (ANCOVA) and baseline adjustment, the mean FSFI score in the drug group was higher compared to the placebo group (adjusted mean difference $=8.70,95 \% \mathrm{CI}=6.58-10.82, P<0.001)($ Table 2 and Figure 2).

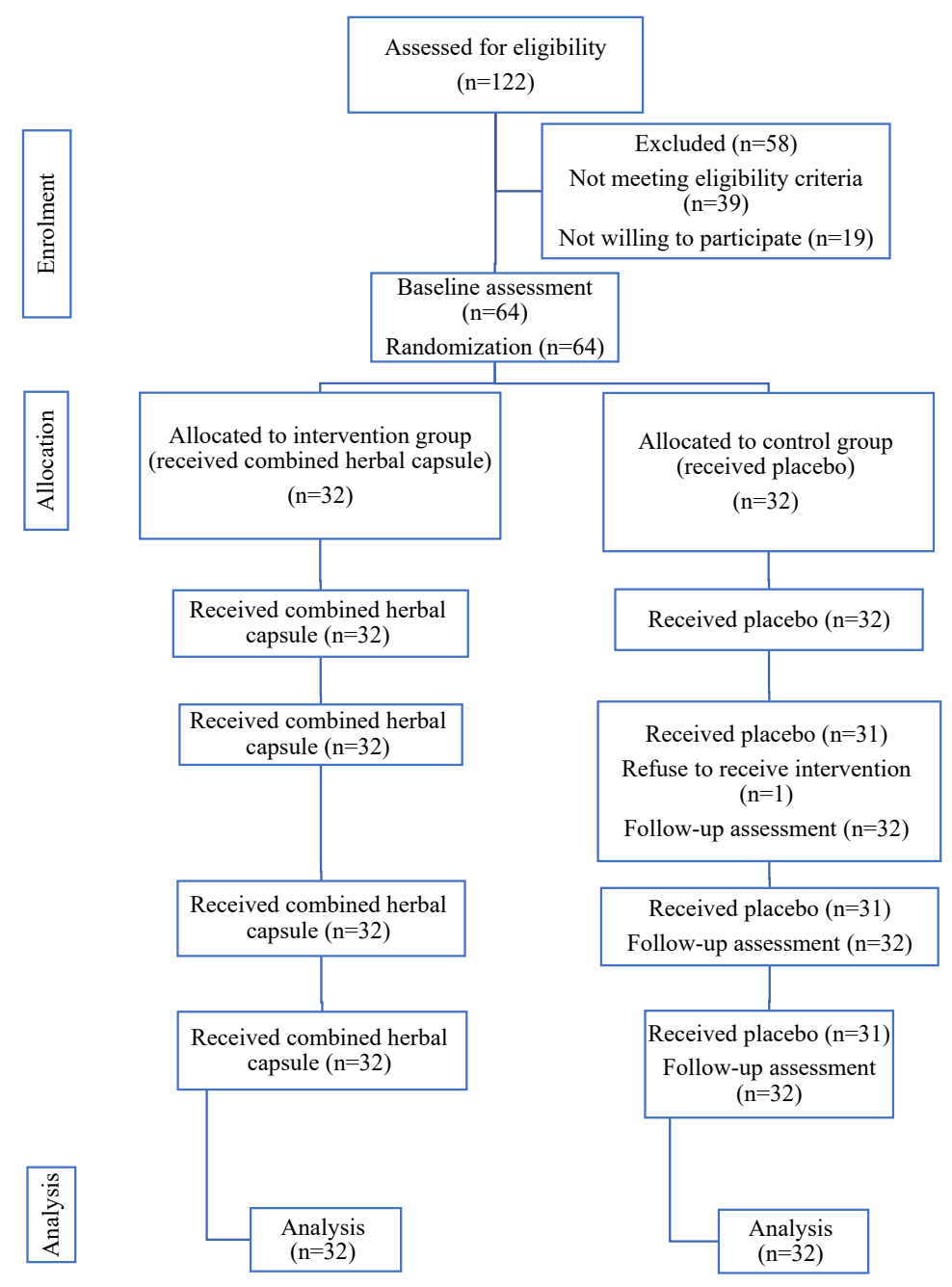

Figure 1. Flowchart of the Study Procedure. 
Table 1. Socio-demographic Characteristics of the Participants in Study Groups

\begin{tabular}{|c|c|c|c|}
\hline Characteristics & $\begin{array}{c}\text { Placebo } \\
(n=32)\end{array}$ & $\begin{array}{c}\text { Combined Herbal } \\
\text { Capsule }(n=32)\end{array}$ & $\boldsymbol{P}$-value \\
\hline Age $(y)^{a}$ & $52.34(4.50)$ & $50.53(4.78)$ & $0.815^{b}$ \\
\hline Occupation & & & $1.00^{c}$ \\
\hline Housewife & $28(87.5)$ & $27(84.4)$ & \\
\hline Employee & $4(12.5)$ & $5(15.6)$ & \\
\hline Economic status & & & $0.341^{\mathrm{d}}$ \\
\hline Adequate & $17(53.1)$ & $14(43.8)$ & \\
\hline Relatively adequate & $12(37.5)$ & $17(53.1)$ & \\
\hline Inadequate & $3(9.4)$ & $1(3.1)$ & \\
\hline Education & & & $0.926^{d}$ \\
\hline Primary school & $16(50.0)$ & $18(56.3)$ & \\
\hline Secondary school & $5(15.6)$ & $4(12.5)$ & \\
\hline High School & $6(18.8)$ & $5(15.6)$ & \\
\hline Diploma & $3(9.4)$ & $1(3.1)$ & \\
\hline University & $2(3.6)$ & $4(12.5)$ & \\
\hline Husband's education & & & $0.836^{d}$ \\
\hline Primary school & $10(31.3)$ & $11(34.4)$ & \\
\hline Secondary school & $6(18.8)$ & $5(15.6)$ & \\
\hline High School & 7 (21.9) & $4(12.5)$ & \\
\hline Diploma & $5(15.6)$ & $6(18.8)$ & \\
\hline University & $4(12.5)$ & $6(18.8)$ & \\
\hline BMI $\left(\mathrm{kg} / \mathrm{m}^{2}\right)^{\mathrm{a}}$ & $27.83(3.61)$ & $27.67(4.16)$ & $0.329^{b}$ \\
\hline Gravidity & & & $0.409^{c}$ \\
\hline$\leq 2$ & $8(25.0)$ & $9(28.1)$ & \\
\hline 3 & $9(28.1)$ & $13(40.6)$ & \\
\hline$\geq 4$ & 15 (46.9) & $10(31.3)$ & \\
\hline Parity & & & $0.162^{\mathrm{e}}$ \\
\hline$\leq 2$ & $8(25.0)$ & $11(34.4)$ & \\
\hline 3 & $10(31.3)$ & $13(40.6)$ & \\
\hline$\geq 4$ & $14(43.8)$ & $8(25.0)$ & \\
\hline Number of children & & & $0.126^{\mathrm{e}}$ \\
\hline$\leq 2$ & $8(25.0)$ & $12(37.5)$ & \\
\hline 3 & $10(31.3)$ & $12(37.5)$ & \\
\hline$\geq 4$ & $14(43.8)$ & $8(25.0)$ & \\
\hline Sense of health & & & $0.101^{d}$ \\
\hline Very bad & $0(0.0)$ & $2(6.3)$ & \\
\hline Bad & $11(34.4)$ & 7 (21.9) & \\
\hline Moderate & $19(59.4)$ & $4(48.4)$ & \\
\hline Good & $2(6.3)$ & $9(28.1)$ & \\
\hline Very good & $0(0.0)$ & $2(6.3)$ & \\
\hline
\end{tabular}

${ }^{a}$ Mean (standard deviation); ${ }^{b}$ Independent $t$ test, ${ }^{\mathrm{c}}$ Fisher exact test;

${ }^{\mathrm{d}}$ Chi-square for trend test; ${ }^{\mathrm{e}} \mathrm{Chi}$-square test.

The data are presented as number (percent) unless specified as ${ }^{\text {a' }}{ }^{\prime}$.

The mean (SD) sexual desire score before the intervention was $2.57 \pm 0.76$ in the drug group and it was $2.56 \pm 0.67$ in the placebo group and no significant difference was observed between groups $(P=1.000)$, but the mean (SD) sexual desire score after the intervention in the drug group was $3.71 \pm 0.80$ and in the placebo group, it was $2.79 \pm 0.76$, based on the results of the ANCOVA test and baseline adjustment, the mean sexual desire score in the drug group was higher compared to the placebo group (0.92; 0.57 to $1.27 ; P<0.001)$.

The mean (SD) sexual arousal score before intervention was $2.41 \pm 0.83$ in the drug group and it was $2.46 \pm 0.56$ in

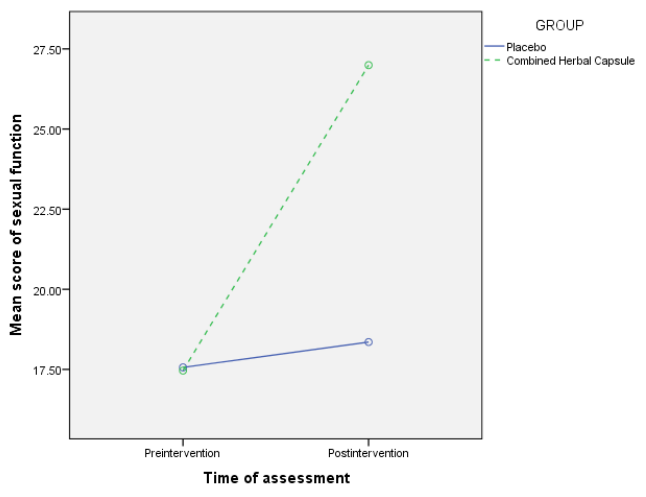

Figure 2. Trend in the Mean Sexual Function Score in Study Groups Before and After the Intervention According to Repeated Measure ANOVA.

the placebo group and there was no significant difference between group $(P=0.793)$, but the mean $(S D)$ sexual arousal score after intervention in the drug group was $3.94 \pm 0.82$ and in the placebo group, it was $2.67 \pm 0.79$. According to the results of the ANCOVA test and baseline adjustment, the mean sexual arousal score in the drug group was significantly higher compared to the placebo group (1.29; 0.95 to $1.63 ; P<0.001)$.

The mean (SD) vaginal lubrication score before the intervention in the drug group was $3.04 \pm 1.13$ and in the placebo group it was $3.03 \pm 0.66$, which was significantly different based on the independent $t$-test and no significant difference was observed between groups $(P=0.968)$, but the mean (SD) lubrication score after intervention in the drug group was $4.70 \pm 1.13$ and in the placebo group, it was $3.22 \pm 0.82$. According to the results of ANCOVA test with adjusting the baseline score, the mean lubrication score in the drug group was significantly higher compared to the placebo group $(1.48,1.03$ to $1.92 ; P<0.001)$.

The mean (SD) orgasm score before the intervention in the drug group was $2.92 \pm 1.08$ and in the control group, it was $2.92 \pm 0.65$. There was no statistically significant difference between groups before the intervention $(P=1.000)$. However, the mean $(\mathrm{SD})$ orgasm score after the intervention was $4.70 \pm 1.07$ in the drug group and it was $3.05 \pm 0.87$ in the placebo group. Based on the results of ANCOVA test with baseline adjustment, the mean orgasm score in the drug group was significantly higher compared to the placebo group $(1.65,1.23$ to $2.07, P<0.001)$.

The mean (SD) satisfaction score before the intervention was $2.69 \pm 1.15$ in the drug group and it was $2.59 \pm 0.87$ in the placebo group, indicating that there was no significant difference between the 2 groups before the intervention $(P=0.699)$. However, mean (SD) satisfaction score after the intervention was $4.73 \pm 0.18$ in the drug group and it was $2.60 \pm 0.18$ in the placebo group. According to the results of ANCOVA test with baseline adjustment, the mean satisfaction score in the drug group was significantly higher compared to the placebo group $(2.31,1.61$ to 2.65 , $P<0.001)$. 
Table 2. Comparison of Mean Sexual Function Score in Study Groups

\begin{tabular}{|c|c|c|c|c|}
\hline Characteristics & $\begin{array}{c}\text { Placebo } \\
(n=32), \text { Mean (SD) }\end{array}$ & $\begin{array}{c}\text { Combined Herbal Capsule } \\
(n=32), \text { Mean (SD) }\end{array}$ & $\begin{array}{c}\text { Mean Difference } \\
(95 \% \mathrm{Cl}) \\
\end{array}$ & $P$-value \\
\hline \multicolumn{5}{|c|}{ Total sexual function score (score 6 to 36 ) } \\
\hline Before intervention & $17.56(3.22)$ & $17.46(5.19)$ & $0.10(-2.06$ to 2.26$)$ & 0.927 \\
\hline 4 weeks after intervention & $18.35(4.51)$ & $26.99(5.31)$ & 8.70 (6.58 to 10.82$)$ & $<0.001$ \\
\hline \multicolumn{5}{|l|}{ Desire (score: 1.2 to 6 ) } \\
\hline Before intervention & $2.56(0.67)$ & $2.57(0.76)$ & $0.18(-0.36$ to 0.36$)$ & 1.000 \\
\hline 4 weeks after intervention & $2.79(0.76)$ & $3.71(0.80)$ & $0.92(0.57$ to 1.27$)$ & $<0.001$ \\
\hline \multicolumn{5}{|l|}{ Arousal (score: 0 to 6 ) } \\
\hline Before intervention & $2.46(0.56)$ & $2.41(0.89)$ & $0.23(-0.31$ to 0.40$)$ & 0.793 \\
\hline 4 weeks after intervention & $2.67(0.79)$ & $3.94(0.82)$ & 1.29 (0.95 to 1.63$)$ & $<0.001$ \\
\hline \multicolumn{5}{|l|}{ Lubrication (score: 0 to 6 ) } \\
\hline Before intervention & $3.03(0.66)$ & $3.04(1.13)$ & $0.23(-0.47$ to 0.45$)$ & 0.968 \\
\hline 4 weeks after intervention & $3.22(0.82)$ & $4.70(1.13)$ & 1.47 (1.03 to 1.92$)$ & $<0.001$ \\
\hline \multicolumn{5}{|l|}{ Orgasm (score: 0 to 6 ) } \\
\hline Before intervention & $2.92(0.64)$ & $2.92(1.08)$ & $0.00(-0.44$ to 0.44$)$ & 1.000 \\
\hline 4 weeks after intervention & $4.70(1.07)$ & $3.05(0.87)$ & 1.65 (1.23 to 2.07$)$ & $<0.001$ \\
\hline \multicolumn{5}{|l|}{ Satisfaction (score 0 to 6 ) } \\
\hline Before intervention & $2.59(0.87)$ & $2.68(1.15)$ & $0.26(-0.61$ to 0.41$)$ & 0.699 \\
\hline 4 weeks after intervention & $2.60(0.18)$ & $4.73(0.18)$ & 2.31 (1.61 to 2.65$)$ & $<0.001$ \\
\hline \multicolumn{5}{|l|}{ Pain (score 0 to 6 ) } \\
\hline Before intervention & $3.99(0.94)$ & $3.82(1.23)$ & $0.27(-0.39$ to 0.71$)$ & 0.557 \\
\hline 4 weeks after intervention & $4.00(0.17)$ & $5.12(0.17)$ & $1.21(0.70$ to 1.71$)$ & $<0.001$ \\
\hline
\end{tabular}

The data are presented as mean (standard deviation).

For comparison of the groups before the intervention, independent $t$ test and after the intervention, ANCOVA with adjusting baseline values were used.

The mean (SD) pain score before the intervention in the drug group was $3.82 \pm 1.23$ and in the placebo group it was $3.99 \pm 0.94$ and there was no significant difference between the 2 groups before the intervention $(P=0.557)$. However, the mean $(\mathrm{SD})$ pain score after the intervention was $5.71 \pm 0.17$ in the drug group and it was $4.00(0.17)$ in the placebo group. According to the results of ANCOVA test and baseline adjustment, the mean pain score in the drug group was significantly higher compared to the placebo group $(1.21,0.70$ to $1.71, P<0.001)$ (Table 2$)$.

Concerning adverse events, $2(6.3 \%)$ women in the placebo group and $6(18.8 \%)$ women in the drug group reported abdominal pain during the intervention.

\section{Discussion}

In this study, the use of the combined herbal capsule significantly increased the total score of sexual function and its subscale in comparison with placebo.

In menopausal period, certain types of sexual dysfunction are constantly increasing due to estrogen deficiency. Vagina and epithelium of urethra are dependent on estrogen and its deficiency cause thinning of epithelium. As a result, atrophic vaginitis causes vaginal dryness symptoms such as itching and painful intercourse $(8,9)$. Vaginal dryness, low sexual desire, and arousal are progressive complications and are associated with hypoestrogenism (8-10).

Anise and fennel in the combined herbal capsule contain phytoestrogens and with estrogen-like properties influence the levels of $\mathrm{LH}$ and FSH hormones, increase sexual desire, resulting in sexual satisfaction and improved sexual behavior in women, and increase vaginal moisture and sexual desire by improving vaginal atrophy (25). Phytoestrogens also cause secretion of neurotransmitters such as endorphin, noradrenaline, and serotonin. These neurotransmitters can be effective in reducing sexual dysfunction (26). Since the combined herbal capsule used in this study was the first to be scientifically examined, the results of other studies using the herbs used in this compound and their impact on sexual function and menopausal complications are reported here.

A similar study was done by Najar et al to assess the effect of Foeniculum vulgare on sexual satisfaction and dyspareunia in postmenopausal women referred to health centers of Ahvaz, Iran. Sixty women with vaginal atrophy were randomly assigned to 2 groups of 30 , who received $5 \%$ fennel vaginal cream and placebo for eight weeks. Sexual satisfaction was assessed using standard Larsson's Sexual Satisfaction Questionnaire. The mean sexual satisfaction score after the intervention in the recipient group of fennel was significantly higher compared to the placebo group and there was a significant decrease in pain intensity during intercourse in the intervention group compared to the placebo group $(P<0.001)$. The possible cause of this effect is the presence of phytoestrogens (18).

In the study conducted by Malakouti et al in Iran, the total score of sexual function and its subscales, except for pain during intercourse, in postmenopausal women 
6 weeks after using aromatherapy with fennel, gerbera, seaweed, orange, lavender and geranium improved $(P<0.001)$. The mechanism of action mentioned in this study is the activation of the olfactory receptor cells by the phytoestrogens in the compounds used and thus secreting neurotransmitters such as endorphin, noradrenaline, and serotonin. These neurotransmitters can be effective in reducing symptoms such as hot flashes, sexual dysfunction and depression (26), which is in agreement with the findings of this study.

In a study by Akhtari et al, the efficacy of Tribulus terrestris extract on 66 women of reproductive age was measured. Four weeks after the intervention, significant differences were observed between 2 intervention and control groups in terms of the total score of sexual function, sexual desire, sexual arousal, vaginal lubrication, and pain during intercourse. It seems that $T$. terrestris contains phytoestrogens which affect levels of LH and FSH hormones and increase sexual desire, resulting in sexual satisfaction and improved sexual behavior in women (25).

In a research conducted in Iran by Yaralizadeh et al on 60 menopausal women (45-65 years old) who were allocated to 2 groups, both placebo and fennel group were requested to use the cream once daily ( 5 gram/day) for eight weeks. In this study, vaginal $\mathrm{pH}$ and vaginal maturation index (VMI) were measured at baseline and 8 weeks after intervention. The number of superficial cells significantly increased in the intervention group compared to the placebo group $(76.1 \pm 1.3$ versus $8.1 \pm 11.8, P<0.001)$ after 8 weeks. The number of intermediate and parabasal cells in the fennel group decreased significantly compared to the control group $(P<0.001)$. The vaginal $\mathrm{pH}$ significantly reduced after 8 weeks in the fennel group compared to the placebo group (100\% versus $7.7 \%$ ). The participants in the fennel group had a VMI of $65-100 \%$ after 8 weeks, while $40.7 \%$ of women in the placebo group had a VMI of $50-64 \%$ (27). The improvement of vaginal atrophy can be considered as a cause of the improvement of sexual function in postmenopausal women in this study.

In a study by Nahidi et al in Iran, the effect of Anise on the improvement and relapse of hot flashes in menopausal women was measured. In this study, 72 postmenopausal women with hot flashes were divided into 2 intervention (recipient of capsules containing $330 \mathrm{mg}$ anise used three times daily) and control (placebo recipient) groups. The intervention was conducted for 4 weeks and the participants were followed up for 4 weeks after intervention. In the drug group, the number and severity of hot flashes before and after treatment were $21.4 \%$ and $56.21 \%$, respectively, followed by $1.6 \%$ and $14.44 \%$ after 4 weeks, respectively. There was no change in the frequency and severity of hot flashes in the control group.

Regarding the presence of phytoestrogens in this plant, it seems that the mechanism of the effect of Anise on hot flashes was through the role of phytoestrogens (28). This study confirmed the presence of phytoestrogens in Anise and its effects on menopausal symptoms, but unfortunately, no research was found to assess the effectiveness of this plant on sexual function.

One of the limitations of the present study was that since more than half of the women had elementary education, they could not have a correct understanding of the questions. However, in order to remove this limitation, the questionnaire was completed through an interview with the participants and in the cases of ambiguity the researcher tried to resolve it. Some of the strengths of the study are the use of triple-blind design and the observance of all clinical trials principles including randomization and allocation concealment. It is suggested that the efficacy of this capsule should be measured on the sexual function among women of reproductive age.

The results of this study showed that the combined herbal capsule could be effective in improving sexual dysfunction in postmenopausal women and reduce the use of hormone therapy in this period. Moreover, the results of this research can be used to complete previous researches and basic information for later researches, including assessing the effectiveness of herbal medicines on sexual function in other age groups.

\section{Conclusions}

In this study, using combined herbal capsules increased the total mean score of sexual function and its subscales in postmenopausal women and it seems that it is able to improve the sexual function of postmenopausal women, Therefore, given the high prevalence of sexual disorders in postmenopausal women, the complications of hormone therapy and the lack of observation of side effects in the combined herbal capsule, this capsule is recommended for treatment of sexual disorders and the reduction of dyspareunia and vaginal dryness in postmenopausal women.

\section{Conflict of Interests}

Authors declare that they have no conflict of interests.

\section{Financial Support}

This study was financially supported by Tabriz University of Medical Sciences.

\section{Acknowledgments}

This study has been extracted from an MSc thesis. The authors appreciate all the participants of this study.

\section{References}

1. Mirghafourvand M, Mohammad-Alizadeh-Charandabi S, Asghari Jafarabadi M, Tavananezhad N, Karkhane M. Predictors of health-related quality of life in Iranian women of reproductive age. Appl Res Qual Life. 2016;11(3):723-737.

2. Mohammad-Alizadeh-Charandabi S, Mirghafourvand M, Khaki-Rostami Z, Malakouti J, Asghari Jafarabadi M, Ghanbari-Homayi S. Sexual dysfunction and help seeking behaviors in newly married men in Sari City: a descriptive. 
J Caring Sci. 2015;4(2):143-153. doi:10.15171/jcs.2015.015

3. Lewis RW, Fugl-Meyer KS, Corona G, et al. Definitions/ epidemiology/risk factors for sexual dysfunction. J Sex Med. 2010;7(4 Pt 2):1598-1607. doi:10.1111/j.17436109.2010.01778.x

4. Ramezani MA, Ahmadi K, Ghaemmaghami A, Marzabadi EA, Pardakhti F. Epidemiology of sexual dysfunction in Iran: a systematic review and meta-analysis. Int J Prev Med. 2015;6:43. doi:10.4103/2008-7802.157472

5. Hashemi S, Ramezani Tehrani F, Simbar M, Abedini M, Bahreinian H, Gholami R. Evaluation of sexual attitude and sexual function in menopausal age; a population based cross-sectional study. Iran J Reprod Med. 2013;11(8):631636.

6. Shifren JL, Schiff I. Reproductive endocrinology. In: Berek JS, ed. Berek and Novak's Gynecology. 15th ed. Philadelphia: Wolters Kluwer Health; 2012:1233-1249.

7. Aghamiri V, Mirghafourvand M, Mohammad-AlizadehCharandabi S, Nazemiyeh H. The effect of Hop (Humulus lupulus L.) on early menopausal symptoms and hot flashes: A randomized placebo-controlled trial. Complement Ther Clin Pract. 2016;23:130-135. doi:10.1016/j.ctcp.2015.05.001

8. Javadivala Z, Merghati-Khoei E, Asghari Jafarabadi M, et al. Efficacy of pharmacological and non-pharmacological interventions on low sexual interest/arousal of peri- and post-menopausal women: a meta-analysis. Sex Relation Ther. 2019;34(2):242-270. doi:10.1080/14681994.2018.144 6515

9. Van Voorhis BJ, Santoro N, Harlow S, Crawford SL, Randolph J. The relationship of bleeding patterns to daily reproductive hormones in women approaching menopause. Obstet Gynecol. 2008;112(1):101-108. doi:10.1097/ AOG.0b013e31817d452b

10. La Marca A, Sighinolfi G, Papaleo E, Cagnacci A, Volpe A, Faddy MJ. Prediction of age at menopause from assessment of ovarian reserve may be improved by using body mass index and smoking status. PLoS One. 2013;8(3):e57005. doi:10.1371/journal.pone.0057005

11. Nastri CO, Lara LA, Ferriani RA, Rosa ESAC, Figueiredo JB, Martins WP. Hormone therapy for sexual function in perimenopausal and postmenopausal women. Cochrane Database Syst Rev. 2013(6):Cd009672. doi:10.1002/14651858.CD009672.pub2

12. Green RR, Santoro N, Allshouse AA, Neal-Perry G, Derby C. Prevalence of Complementary and Alternative Medicine and Herbal Remedy Use in Hispanic and NonHispanic White Women: Results from the Study of Women's Health Across the Nation. J Altern Complement Med. 2017;23(10):805-811. doi:10.1089/acm.2017.0080

13. Poluzzi E, Piccinni C, Raschi E, Rampa A, Recanatini M, De Ponti F. Phytoestrogens in postmenopause: the state of the art from a chemical, pharmacological and regulatory perspective. Curr Med Chem. 2014;21(4):417-436.

14. Saghafi N, Ghazanfarpour M, Sadeghi R, et al. Effects of phytoestrogens in alleviating the menopausal symptoms: A systematic review and meta-analysis. Iran J Pharm Res. 2017;16(Suppl):99-111.

15. Mense SM, Hei TK, Ganju RK, Bhat HK. Phytoestrogens and breast cancer prevention: possible mechanisms of action.
Environ Health Perspect. 2008;116(4):426-433. doi:10.1289/ ehp. 10538

16. Nazem E. Tooba pharmacopoeia. Tehran: Andishehavar; 2010.

17. Moghimian M, Abtahi-Evari SH, Shokoohi M, Amiri M, Soltani M. Effect of Syzygium aromaticum (clove) extract on seminiferous tubules and oxidative stress after testicular torsion in adult rats. Physiology and Pharmacology. 2017;21(4):343-350.

18. Najar S, Yaralizadeh M, Abedi P, Namjooyan F, Saki Malehi O. Effect of Fennel vaginal cream on dysparonia and sexual satisfaction among postmenopausal women: A doubleblind randomized controlled trial. The Iranian Journal of Obstetrics, Gynecology and Infertility. 2015;18(171):8-16. [Persian].

19. Rahimikian F, Rahimi R, Golzareh P, Bekhradi R, Mehran A. Effect of Foeniculum vulgare Mill. (fennel) on menopausal symptoms in postmenopausal women: a randomized, triple-blind, placebo-controlled trial. Menopause. 2017;24(9):1017-1021. doi:10.1097/gme.0000000000000881

20. Nassiri-Asl M, Hosseinzadeh H. Review of the Pharmacological Effects of Vitis vinifera (Grape) and its Bioactive Constituents: An Update. Phytother Res. 2016;30(9):1392-1403. doi:10.1002/ptr.5644

21. Mohammadi KH, Heydari M, Faghihzadeh S. The female sexual function index (FSFI): validation of the Iranian version. Payesh. 2008;7(3): 269-278.

22. Schurger N, Klein E, Hapfelmeier A, Kiechle M, Paepke D. Demand for integrative medicine among women in pregnancy and childbed: a German survey on patients' needs. BMC Complement Altern Med. 2018;18(1):187. doi:10.1186/s12906-018-2249-y

23. Nazarpour S, Simbar M, Ramezani Tehrani F, Alavi Majd H. Relationship between Sexual Function and Quality of Life in Post-menopausal Women. Journal of Mazandaran University of Medical Sciences. 2016;26(143):90-98. [Persian].

24. Rosen R, Brown C, Heiman J, et al. The Female Sexual Function Index (FSFI): a multidimensional self-report instrument for the assessment of female sexual function. J Sex Marital Ther. 2000;26(2):191-208.

25. Akhtari E, Raisi F, Keshavarz M, et al. Tribulus terrestris for treatment of sexual dysfunction in women: randomized double-blind placebo - controlled study. Daru. 2014;22:40. doi:10.1186/2008-2231-22-40

26. Malakouti J, Farshbaf Khalili A, Asghari Jafarabadi M, Jabbari F. Effect of combined inhaler aromatherapy on sexual function in postmenopausal women: A randomized controlled trial. The Iranian Journal of Obstetrics, Gynecology and Infertility. 2016;19(1-2):9-15. [Persian].

27. Yaralizadeh M, Abedi P, Najar S, Namjoyan F, Saki A. Effect of Foeniculum vulgare (fennel) vaginal cream on vaginal atrophy in postmenopausal women: A double-blind randomized placebo-controlled trial. Maturitas. 2016;84:7580. doi:10.1016/j.maturitas.2015.11.005

28. Nahidi F, Kariman N, Simbar M, Mojab F. The study on the effects of Pimpinella anisum on relief and recurrence of menopausal hot flashes. Iran J Pharm Res. 2012;11(4):10791085. provided the original work is properly cited. 